Original Research Paper

\title{
Comparison of Edge Detection Techniques Applied in the Identification of Centerline Segregation on Steel Slabs
}

\author{
${ }^{1}$ Luciene Coelho Lopez Queiroz and ${ }^{2}$ André Riyuiti Hirakawa \\ ${ }^{1}$ Department of Industrial Engineering, Usinas Siderúrgicas de Minas Gerais-Usiminas, Cubatão, Brazil \\ ${ }^{2}$ Department of Electrical Engineering, Polytechnic School University of São Paulo-USP, São Paulo, Brazil
}

\author{
Article history \\ Received: 18-04-2014 \\ Revised: $10-05-2014$ \\ Accepted: 29-08-2015 \\ Corresponding Author: \\ Luciene Coelho Lopez Queiroz \\ Department of Industrial \\ Engineering, Usinas \\ Siderúrgicas de Minas Gerais- \\ Usiminas, Cubatão, Brazil \\ Email: luciene.lopez@usiminas.com
}

\begin{abstract}
The method Baumann, or Sulphur print as it is also known, is one of the tools used to evaluate the operating conditions of continuous casting machine and quality control of the production of steel slabs. The correct evaluation of centerline segregation severity, analyzed in Sulphur print, is essential to control this process. However, given the classification complexity between the different severity levels, the classification process becomes dependent on experts experience and knowledge. In the light of human interference in this analysis, differences on classification results are possible, since the activity is manual and occasionally an expert can be stricter than others during the classification process. The evaluation of this scenario has motivated the search for the development of computational resources able to identify and classify the defect of centerline segregation. The edge detection techniques are applied to identify the centerline segregation, to reduce the amount of data to be processed and discard information considered irrelevant image while preserving the structural features of the regions of interest that will be used later to classify the severity of the defect. This paper presents a comparison between Sobel, Laplacian of Gaussian and Canny edge detection methods applied to the digital images of the samples generated during Baumann method for identifying the defect of centerline segregation.
\end{abstract}

Keywords: Edge Detection, Digital Image Processing, Centerline Segregation, Baumann's Method

\section{Introduction}

Among the different processes that compound an integrated steel plant, stands out the continuous casting steel process. At this stage, the steel is converted from liquid to solid state through the Continuous Casting Machine (CCM), yielding semi-finished products such as profiles, slabs, blooms or billets.

The process of steel solidification in the continuous casting occurs towards the edges to the core of the strand due to the contact with the mold cooled walls, water sprays and radiation heat loss to the environment. This heat transfer makes steel gradually solidify creating areas where the material can be found in their solid and liquid states. Voort (1999) explains that during the solidification phenomenon phosphorus and sulfur are rejected from solid to liquid zone because the solubility of solid sulfur in iron is very low, nearly all the sulfur is precipitated as sulfide inclusions. Thus, the last solidified regions of slab have a high concentration of impurities, which directly influences the quality of material produced.

The quality control of the produced material and the operating conditions of the CCM are performed by Baumann's method, or Sulfur print (S-Print) as it is also known. Faco (2005) points out that the purpose of this essay is to identify and classify internal defects such as internal cracks (horizontal, vertical and corner), centerline segregation, alumina inclusions and porosity, which would prejudice the implementation of the produced material as was intended. Through the analysis of the results is possible to determine the corrective actions necessary to ensure adequate operating conditions of the CCM, besides the quality of the manufactured products.

Baumann's method can be considered a graphical representation of the internal quality of casting material and as it is widespread applied, is subject to standardization by different regulatory bodies internationally recognized. Among the norms that standardize the procedures for 
conducting the Baumann's method stand out ISO (1979), BS (1982) and ASTM (2008).

In general, on the steel plants such internal defects are visually rated by experts by comparing the Sulphur print sample with pre-established grading standards. Furtado (2007) explains that these standards are drawn up by steel producing companies and among the best known, highlight the Nippon Steel Corporation (NSC) and Mannesmann standards.

However, given the classification complexity between the different severity levels of each defect, the classification process becomes dependent on experts experience and knowledge. In the light of human interference in this analysis, differences on classification results are possible, since the activity is completely manual and occasionally an expert can be stricter than others during the classification process.

The evaluation of this scenario has motivated the search for the development of computational resources able to identify and classify the defect of centerline segregation by processing digital images of Sulphur print sample. This paper presents a comparison between different edge detection techniques applied in the identification of image region of interest (centerline segregation) on Sulphur print sample.

\section{Centerline Segregation}

Ghosh (2001) points out that the presence of centerline segregation negatively influences the performance of the rolled product because modify the physical and mechanical properties of the slabs. As higher is the centerline segregation, higher its impact on the quality of the final product.

Rizzo (2006) explains that the centerline segregation is a defect that occurs in the last region solidified of the slab. By having this feature, this defect is concentrated in the central area of the product. According Voort (1999) the latest regions solidified of the slab have a high concentration of harmful impurities to the quality of steel, among which stands out the sulfur. The centerline segregation is identified as the region where a high concentration of dark or black spots occurs horizontally near the center of the sample. In Fig. 1, the centerline segregation can be observed in the Sulphur print sample.

According to Carvalho (1988) due to the phenomenon of steel solidification, this defect becomes inherent to continuous casting process. Thus, the centerline segregation can hardly be eliminated from the slab. However, a large accumulation of impurities may compromise the quality of the material produced, making the systematic monitoring of the level of segregation essential to the process.

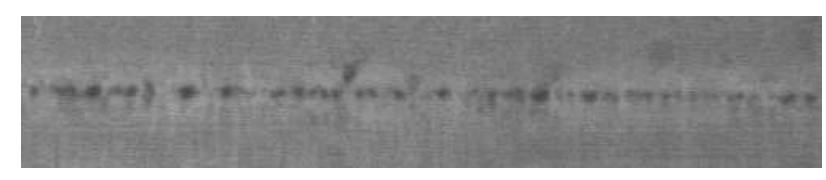

Fig. 1. Centerline segregation

The correct classification of the severity degree of centerline segregation is fundamental in monitoring the continuous casting process. In order to correctly identify the centerline segregation through Sulphur print digital image processing, the image segmentation step is essential.

Pedrini and Schwartz (2008) define the segmentation of an image as the act of partition it into different regions according to certain properties.

The basic task of the segmentation step is to divide an image into its meaningful units, in other words, the objects of interest that comprise it (Marques and Vieira, 1999). This feature makes the segmentation be considered, among all stages of image processing, the most critical step of data processing, since at this stage the regions of interest, which will be used in the next processing steps are defined.

Among the techniques for segmentation, stands out the techniques for edge detection, which are based on the approach of analyses of discontinuities between pixels.

\section{Edge Detection Technique}

The purpose of the edge detection techniques is to reduce the amount of data to be processed and discard information considered irrelevant image while preserving the structural features of the regions of interest that will be used in subsequent steps of image processing. Pedrini and Schwartz (2008) define the edge as the boundary or border between two regions with relatively distinct gray level properties. These characteristics are used, for example, to distinguish between an object of interest, the background of an image.

Pedrini and Schwartz (2008) argue that edge detection is essentially the operation of identifying significant local changes in gray level image and these changes can be described by the concept of derivative. According Shrivakshan and Chandrasekar (2012) there are several techniques for edge detection, but they can be grouped into two categories: Gradient and Laplacian.

In the gradient method the edges are detected through maximum and minimum of the first order derivative of the image. On the other hand, in the Laplacian method, the edges are detected through the zero crossing of the second order derivative. Both approaches can be approximated by convolution masks.

\section{Sobel}

The Sobel operator to edge detection uses two matrices of size $3 \times 3$ as mask in order to calculate an 
approximation of the gradient of the image intensity function. The first matrix $G x$ estimates the gradient in $x$ axis direction while the second $G y$ estimates the gradient in the direction of the $y$ axis (Ma et al., 2010). Each mask is convolved with image $N$, as shown in Equation 1 and 2 , to calculate the approximate derived from changes in horizontal and vertical intensity ( $G x$ and $G y)$ :

$$
\begin{aligned}
& G_{x}=\left[\begin{array}{ccc}
-1 & 0 & 1 \\
-2 & 0 & 2 \\
-1 & 0 & 1
\end{array}\right] * N \\
& G_{y}=\left[\begin{array}{ccc}
1 & 2 & 1 \\
0 & 0 & 0 \\
-1 & -2 & -1
\end{array}\right] * N
\end{aligned}
$$

Then, for each image point, the gradient magnitude is calculated according to Equation 3 by combining partial results of the horizontal and vertical gradient obtained in previous calculations (Deng et al., 2011):

$|G|=\sqrt{G_{x}^{2}+G_{y}^{2}}$

\section{Laplacian of Gaussian}

The Laplacian of Gaussian (LOG) operator or as it is also known Marr-Hildreth operator was first proposed by Marr-Hildreth in 1980 based on his research into the human visual system (Zhao and deSilva, 1998). In this method the Gaussian filter is matched to Laplacian.

First, the Gaussian filter is applied to the image in order to minimize the noise, which increases the sensitivity and enhances the Laplacian edge detection process. The Laplacian, highlights the regions where there is an abrupt change in intensity, which is used to identify edges. The Laplacian operator is defined by Equation 4 (Shrivakshan and Chandrasekar, 2012):

$$
\operatorname{Laplace}(f)=\frac{\partial^{2} f}{\partial x^{2}}+\frac{\partial^{2} f}{\partial y^{2}}
$$

\section{Canny}

Canny (1986) described a computational approach to edge detection, later called the Canny operator. In their method, Canny established that an edge detector must essentially satisfy three basic performance criteria: Good detection, good location and good response. Cheng et al. (2008) described, in other words, that the Canny operator aims reduce the error rate while minimizing the number of false positives (pixels that do not represent edges detected) and false negatives (pixels representing edges, but which are not detected); minimize the distance between the detected edge and the real edge of the image processed; minimize the likelihood of multiple responses to a single edge.

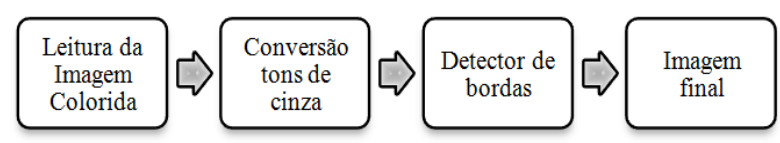

Fig. 2. Algorithm steps

The Canny method can be divided into four steps (Shrivakshan and Chandrasekar, 2012):

- Smoothing of the input image by applying the Gaussian filter, aimed at removing irrelevant details like texture elements and noise

- Determining the magnitude and direction of the gradient for each pixel of the smoothed image

- Application of non-maximum for the gradient magnitude suppression technique. This step aims at removing the pixels whose values are not local maxima, in the direction perpendicular to the edge, providing the best location and thinning the edge

- Application of thresholding technique, using two thresholds (upper and lower), aimed at removing weak edges

\section{Implementation and Comparison}

Three edge detection techniques have been selected to perform a comparative identification of the centerline segregation on Sulphur print images (Sobel and Canny Laplacian of Gaussian). The proposed approach shown in Fig. 2, initially consists in reading the Sulphur print image sample to be processed by the algorithm. Then the image is converted to grayscale range and finally the edge detection techniques are applied to give the final image with the edges and boundaries detected.

The implementation of the algorithm was done in $\mathrm{C}++$ using Visual C++ 2010 Express Edition (Microsoft, 2010) compiler. All edges detectors were implemented using the OpenCV library version 2.4.2 (OpenCV, 2012). The algorithm is executed individually and analyzed for each technique.

\section{Results}

The edge detection techniques were applied to a segment of the Sulphur print sample image and the results may be seen in Fig. 3 .

In Sobel method, the result shows thick and distorted edges, which contributes to the loss of information about the structure of the centerline segregation, which could compromise the subsequent stages of image processing. Another important aspect is the excessive occurrence of false positives, in other words, noises that were identified as edges in the image. 
(a)

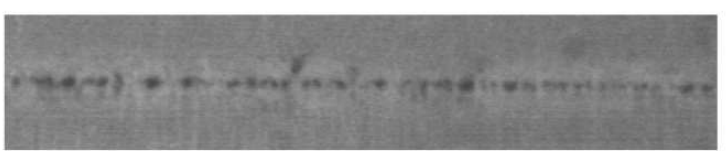

(b)

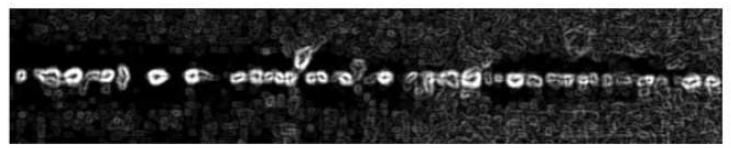

(c)

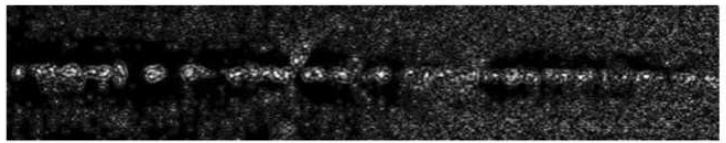

(d)

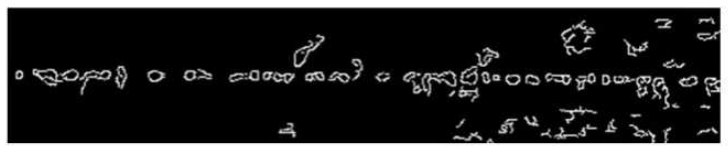

Fig. 3. Implementation of edge detection, 4-a. Original image; 4-b. Sobel result; 4-c. Laplacian of Gaussian result; 4-d. Canny result

In the application of Laplacian of Gaussian method, the test result shows the loss of important data for identification of the structure of the centerline segregation and the edges were not properly identified, complicating the identification of regions of interest of the image.

For the Canny edge detector, the lower threshold of 17 and upper 37 was adopted. By adopting these values, the edges were identified and noise in the image has been almost eliminated, while structural characteristics of centerline segregation were preserved. Even with the occurrence of false positives in the image (detection of edges where there are not effectively), in the Canny method, a smaller number of pixels corresponding to noise, have been identified as "edge" compared to the Sobel method.

Note that in the Canny method the correct setting of thresholds is fundamental for obtaining satisfactory results. The adoption of a very large threshold may cause the loss of important information. In contrast, the adoption of a very small threshold may result in excessive identification of unnecessary information (noise) as edges. The challenge in using this method is just to find a (not too small or large) generic threshold that presents satisfactory results with different images.

\section{Discussion}

The claim that an edge detection technique is better or worse than another cannot be performed. The analysis that must be performed is about which technique has the best result for a particular type of application. The best result should be evaluated according to its ability to preserve the structural elements of the regions of interest and noise reduction in the image. The balance between these two aspects indicates that a technique has a satisfactory performance for a specific application.

In specific application of centerline segregation classification, the detection of the regions of interest preserving the structural elements is critical because are the features extracted from structural elements that will later be used to classify the centerline segregation according with the different degrees of severity. Due to this, as better the balance between noise reduction and structural elements preservation, better could be the assertiveness to classify the defect, since irrelevant image information have been eliminated at this stage.

Among the edge detection techniques analyzed for the identification of centerline segregation on Sulfur print images, it was observed that the results obtained by the Canny method stood out compared to the results obtained by the Sobel and Laplacian of Gaussian methods due to the balance achieved between these two aspects.

\section{Conclusion}

Edge detection is the first step in the border delimitation and identification of objects, so it is essential to know the characteristics of different methods of edge detection. In this study was presented a brief review of some edge detection techniques based on the approach of discontinuities analysis between pixels.

The techniques analyzed were applied to the problem of identifying the defect of centerline segregation on Sulphur print samples. The comparison of the analyzed methods shows positive results in applying these techniques in identifying the centerline segregation.

Additionally, it was observed that with the thresholds adopted, the Canny method showed better results in detecting the edges of the centerline segregation when compared to the Sobel and Laplacian of Gaussian methods.

The challenge in adopting Canny method is to find a (not too small or large) generic threshold that presents satisfactory results with different images. This characteristic allied to the results obtained on the comparison performed, encourage further studies about the application of the Canny method using adaptive thresholds to improve the identification and classification of centerline segregation on Sulphur print images.

\section{Funding Information}

The authors of this paper have no financial funding to report.

\section{Author's Contributions}

This paper is the result of the full and equal collaboration of all the authors. 


\section{Ethics}

The authors confirm that this article is original and all of the authors have read and approved the manuscript.

\section{References}

ASTM, 2008. ASTM E1180-08: Standard practice for preparing sulfur prints for macrostructural evaluation. Am. Society Testing Mater. DOI: $10.1520 / \mathrm{E} 1180-08$

BS, 1982. BS 6285: The macrographic examination of steel by sulphur print (Baumann method). British Standard, London.

Canny, J.F., 1986. A computational approach to edge detection. IEEE Trans. Patt. Analysis Machine Intellig., 8: 679-698.

DOI: 10.1109/TPAMI.1986.4767851

Carvalho, J.L., 1988. Lingotamento Contínuo de Placas de Aço. São Paulo: Associação Brasileira de Metais.

Cheng, J., R. Xue, W. Lu and R. Jia, 2008. Segmentation of medical images with Canny operator and GVF snake model. Proceeding of the 7th World Congress on Intelligent Control and Automation, Jun. 25-27, IEEE Xplore Press, Chongqing, pp: 1777-1780. DOI: 10.1109/WCICA.2008.4593191

Deng, C., W. Ma and Y. Yin, 2011. An edge detection approach of image fusion based on improved Sobel operator. Proceedings of the 4th International Congress on Image and Signal Processing, Oct. 1517, IEEE Xplore Press, Shanghai, 3: 1189-1193. DOI: 10.1109/CISP.2011.6100499

Faco, R.J., 2005. Lingotamento Contínuo do Aço. In: Siderurgia Para Não Siderurgistas, Mourão, M.B. (Ed.), São Paulo, Associação Brasileira de Metalurgia e Materiais, pp: 147-147.

Furtado, H.S., 2007. Controle da Qualidade de Placas. In: Lingotamento Contínuo De Placas, Valadares C.A.G. (Ed.), São Paulo, Associação Brasileira de Metalurgia e Materiais, pp: 186-186.
Ghosh, A., 2001. Segregation in cast products. Sadhana, 26: 5-24. DOI: 10.1007/BF02728476

ISO, 1979. ISO 4968: Steel-Macrographic examination by sulphur print (Baumann method). International Standard Organization, Genebra.

Ma, C., W. Gao, L. Yang and Z. Liu, 2010. An improved Sobel algorithm based on median filter. Proceedings of the 2nd International Conference on Mechanical and Electronics Engineering, Aug. 1-3, IEEE Xplore Press, Kyoto, pp: 88-92. DOI: 10.1109/ICMEE.2010.5558590

Marques, O.F. and H.N. Vieira, 1999. Processamento Digital De Imagens. 1st Edn., Brasport, ISBN-10: 8574520098, pp: 406.

Microsoft, 2010. Visual C++ Express.

OpenCV, 2012. Open Source Computer Vision.

Pedrini, H. and W.R. Schwartz, 2008. Análise de imagens digitais: Princípios, algoritmos e aplicações. São Paulo: Thomson Learning.

Rizzo, E.M.S., 2006. Introdução Aos Processos De Lingotamento Dos Aços. 1st Edn., ABM, São Paulo, ISBN-10: 8586778958, pp: 158.

Shrivakshan, G.T. and C. Chandrasekar, 2012. A comparison of various edge detection techniques used in image processing. Int. J. Comput. Sci., 9: 269-276.

Voort, G.F.V., 1999. Metallography: Principles and Practices. 1st Edn., ASM International, New York, ISBN-10: 0871706725, pp: 752.

Zhao, F. and C.J.S. deSilva, 1998. Use of the Laplacian of Gaussian operator in prostate ultrasound image processing. Proceedings of the 20th Annual International Conference of the IEEE Engineering in Medicine and Biology Society, 29 Oct-1 Nov., IEEE Xplore Press, Hong Kong, pp: 812-815. DOI: $10.1109 /$ IEMBS.1998.745557 